\title{
Strafbares Streben nach Freiheit?
}

Urteil «Hassan Kiko» des Obergerichts des Kantons Zürich SB170278 vom 13. Oktober 2017

\author{
Marc Thommen / Micha Nydegger *
}

Die Autoren nehmen den vom Obergericht des Kantons Zürich am 13. Oktober 2017 bestätigten Schuldspruch gegen Hassan Kiko wegen Anstiftung zum Entweichenlassen von Gefangenen (Art. 24 Abs. 1 i.V.m. Art 319 StGB) zum Anlass, die Strafbarkeit der Anstiftung zur eigenen Freilassung einer kritischen Betrachtung zu unterziehen. Während Hassan Kiko die Gefängniswärtern Magdici zwar «zur Tat bestimmt» und damit angestiftet hat, ist die formalistische Abgrenzung von Art. 305 und 285 StGB anhand vermeintlich verschiedener Rechtsgüter abzulehnen. In Anlehnung an den Fall Dammann sabotiert der Gefangene, welcher eine Wärterin bittet, ihn freizulassen, nicht das Vertrauen in die Justiz, sondern reklamiert seine «excusable aspiration à la liberté». Triebfeder ist der Selbstschutz, es liegt mithin reine Selbstbegünstigung vor.

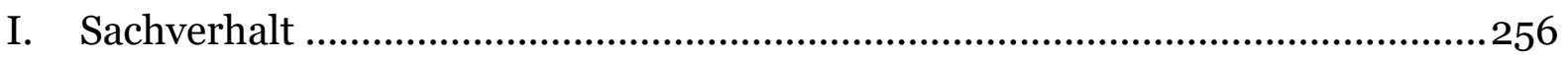

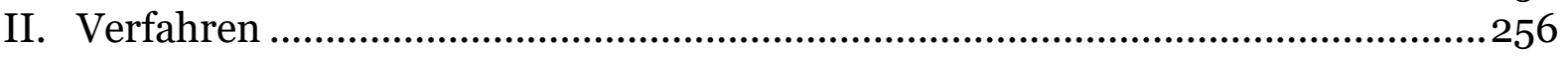

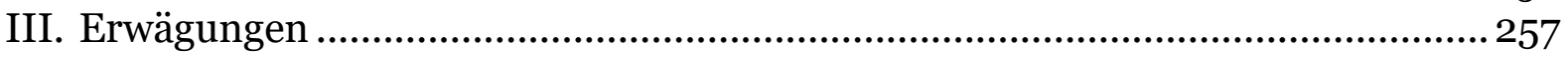

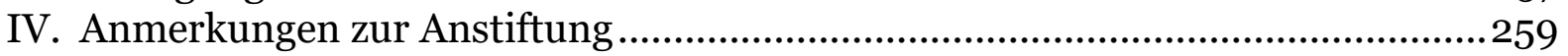

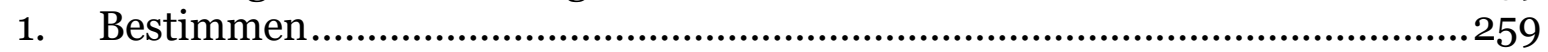

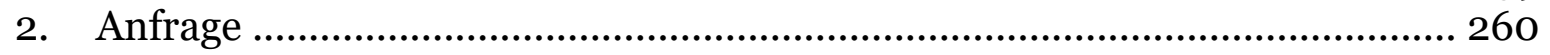

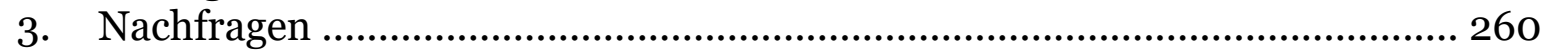

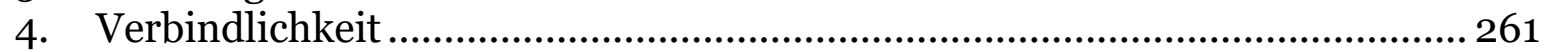

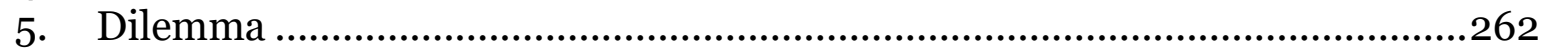

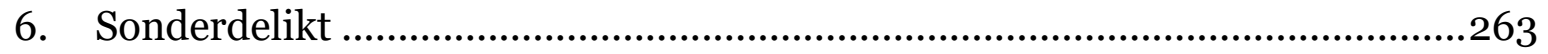

V. Anmerkungen zur Selbstbegünstigung ....................................................264

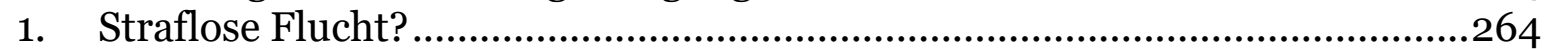

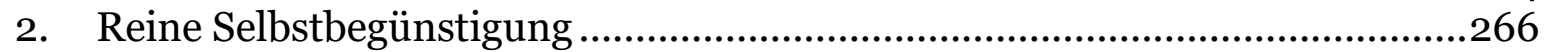

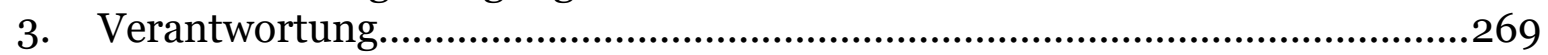

Zitiervorschlag: Marc Thommen/Micha Nydegger, Strafbares Streben nach Freiheit?, in: sui-generis 2018, S. 255

URL: $\quad$ sui-generis.ch/72

DOI: https://doi.org/10.21257/sg.72

* Marc Thommen (marc.thommen[at]uzh.ch), Prof. Dr. iur. LLM (Cantab), ist Inhaber eines Lehrstuhls für Strafrecht und Strafprozessrecht an der Universität Zürich. Micha Nydegger (micha.nydegger[at]kg.gr.ch), Dr. iur., Rechtsanwalt, ist Aktuar/Gerichtsschreiber am Kantonsgericht von Graubünden und Lehrbeauftragter für Strafrecht an der Universität Luzern. Die Autoren danken Luca Ranzoni, cand. MLaw, für seine Hilfe bei der Recherche und der Zusammenstellung des Sachverhalts sowie Dominik Elser, MLaw, für sein sehr sorgfältiges Lektorat. 


\section{Sachverhalt}

1 Es geht um einen Fall, der vor allem in der Schweizer Boulevardpresse für grosses Aufsehen sorgte:

«Gefängniswärterin Angela Magdici verliebt sich in den verurteilten Vergewaltiger Hassan Kiko und verhilft ihm zur Flucht. Wochenlang sucht ganz Europa nach den beiden und fasst sie schliesslich in Italien. Für die ehemalige Aufseherin hat die Tat ein gerichtliches Nachspiel. 15 Monate bedingt, so das Urteil.»1

2 Auch für Hassan Kiko hatte die Flucht juristische Konsequenzen. Am 7. Juli 2016 erhob die Staatsanwaltschaft beim Bezirksgericht Dietikon Anklage gegen ihn wegen Anstiftung zum Entweichenlassen von Gefangenen im Sinne von Art. 319 i.V.m. Art. 24 StGB. Der (unbestrittene) Sachverhalt wurde vom Obergericht wie folgt zusammengefasst: Der Beschuldigte hat am 3. Dezember 2015 Gefängnisaufseherin «B.»², zu welcher er in zahlreichen Gesprächen ein enges persönliches Verhältnis aufgebaut hatte, gebeten, ihm bei der Flucht aus dem Gefängnis behilflich zu sein. Er hatte diese Bitte in den folgenden Monaten mehrfach wiederholt, bis er B. schliesslich überzeugen konnte. Am 7. Februar 2016 fasste B. dann den Entschluss, der Bitte des Beschuldigten nachzukommen, und setzte diesen am 8. Februar 2016 in die Tat um. Der Beschuldigte hat in B. den Entschluss geweckt, ihn aus dem Gefängnis entweichen zu lassen, indem er sie dazu überredet hat, obwohl er um ihre Stellung als Gefängnisaufseherin und der damit verbundenen Aufgaben wusste. 3

\section{Verfahren}

3 Das Bezirksgericht stellte das Verfahren gestützt auf Art. 329 Abs. 4 StPO zunächst mit der Begründung ein, dass eine Bestrafung nach Art. 319 StGB die Straflosigkeit der Selbstbegünstigung unterlaufen würde und das in der Anklage umschriebene Verhalten damit keinen Straftatbestand erfülle. 4

4 Auf Beschwerde der Staatsanwaltschaft hin hob das Obergericht des Kantons Zürich die erstinstanzliche Einstellungsverfügung auf und wies das Verfahren an das Bezirksgericht zurück mit der Aufforderung, die Strafbarkeit des Beschuldigten materiell zu beurteilen. 5

5 In neuer Besetzung sprach das Bezirksgericht Dietikon den Beschuldigten mit Urteil vom 23. Mai 2017 der Anstiftung zum Entweichenlassen von Gefangenen im Sinne von Art. 319 i.V.m. Art. 24 StGB schuldig und bestrafte ihn mit einer unbedingten Freiheitsstrafe von sechs Monaten.

6 Am 24. Mai 2017 liess B. Berufung anmelden. Mit Urteil vom 13. Oktober 2017 bestätigte das Obergericht des Kantons Zürich den erstinstanzlichen Schuldspruch und fällte ebenfalls eine sechsmonatige unbedingte Freiheitsstrafe aus.
Zusammenfassung von telezueri.ch (Gefängnisflucht von Angela Magdici und Hassan Kiko).

2 So die angesichts der unterdessen erlangten Prominenz der Beteiligten sinnbefreite Anonymisierung durch das Obergericht.
3 Vgl. E. II.1.2.

4 Siehe E. I.1.2.

E. I.1.3. 
Das Urteil wurde nicht an das Bundesgericht weitergezogen. Es ist am 15. Januar 2018 in Rechtskraft erwachsen. ${ }^{6}$

\section{Erwägungen}

7 2.1.2. Nach Art. 319 StGB macht sich (u.a.) derjenige Beamte strafbar, der einem Gefangenen zur Flucht behilflich ist oder ihn entweichen lässt. Der Anstiftung zu einem Entweichenlassen eines Gefangenen macht sich demgegenüber gemäss Art. 24 StGB diejenige Person schuldig, die einen Gefängnisbeamten zu einer solchen von diesem verübten Tat vorsätzlich bestimmt hat. Derweil die Tat gemäss Art. 319 StGB ausschliesslich von Beamten begangen werden kann, kann die Anstiftung zu diesem Sonderdelikt grundsätzlich durch jedermann vorgenommen werden.

Zwischen dem motivierenden Verhalten des Anstifters und dem Tatentschluss des Angestifteten muss ein Kausal- bzw. Motivationszusammenhang bestehen. Nicht erforderlich ist, dass beim Anzustiftenden Widerstände zu überwinden wären. Selbst eine zur Tat bereits geneigte Person kann angestiftet werden. Als Anstiftungsmittel kommt jedes motivierende Tun in Frage, alles, was im andern den Handlungsentschluss hervorrufen kann. So ist auch eine blosse Bitte, eine Anregung oder konkludente Aufforderung taugliches Anstiftungsmittel. In subjektiver Hinsicht erfordert die Strafbarkeit wegen Anstiftung Vorsatz, welcher sich zum einen auf die Herbeiführung des Tatentschlusses und zum andern auf die

Mündliche, an dieser Stelle zu verdankende Auskunft vom 14. Mai 2018 von Bruno Bosshard, Weibel der II. Strafkammer des Obergerichts des Kantons Zürich.
Ausführung der Tat durch den Angestifteten beziehen muss. Der Anstifter muss also zumindest in Kauf nehmen, dass der Angestiftete infolge seines Verhaltens eine Handlung begehen wird, welche die objektiven und subjektiven Merkmale eines bestimmten Straftatbestandes erfüllt. Die Tat, zu welcher angestiftet wird, muss ihrerseits eine Vorsatztat sein. [...]

9 2.2.2. Wer jemanden der Strafverfolgung bzw. dem Straf- oder Massnahmenvollzug entzieht, macht sich der Begünstigung nach Art. 305 Abs. 1 StGB strafbar. Straflos bleibt allerdings die blosse Selbstbegünstigung. Nicht strafbar ist deshalb, wer sich selber der Strafverfolgung oder dem Vollzug einer Sanktion entzieht (BGE 115 IV 230 E. 1; 124 IV 127 E. 3.b/aa). Gleiches gilt, wenn der Verfolgte/Verurteilte einen Dritten dazu anstiftet oder diesem dabei Hilfe leistet (BGE 115 IV 230 E. 2). Straffrei ist aber lediglich die reine Selbstbegünstigung. Dass derjenige, der sich der Strafverfolgung oder dem Vollzug einer Strafe entzieht, nicht nach Art. 305 StGB bestraft wird, bedeutet nach der Rechtsprechung des Bundesgerichts nicht, dass er in jedem Fall in den Genuss der Straffreiheit kommt. Denn seine Handlung kann zusätzlich einen anderen Straftatbestand erfüllen.

10 Dies ist beispielsweise dann der Fall, wenn die Flucht - vom Flüchtigen beabsichtigt - bewirkt, dass ein Beamter an der Vornahme einer ihm obliegenden Amtshandlung gehindert wird. So macht sich gemäss der Rechtsprechung des Bundesgerichts etwa der Verurteilte nach Art. 286 StGB strafbar, welcher, um dem mit seiner Überführung ins Gefängnis betrauten Polizeibeamten zu entkom- 
men, die Flucht ergreift und jenen derart an der Erfüllung seines Auftrages hindert. Die Gründe, die in einem solchen Fall der Anwendung von Art. 305 StGB entgegenstehen, gelten im Hinblick auf Art. 286 StGB nicht (BGE 124 IV 127 E. 3.b/bb). Dies deshalb, da zu berücksichtigen ist, dass nach der Systematik des Strafgesetzbuches die beiden Tatbestände der Hinderung einer Amtshandlung und der Begünstigung verschiedene Rechtsgüter schützen, nämlich Art. 286 StGB den Schutz der öffentlichen Gewalt und Art. 305 StGB den Schutz der Strafrechtspflege. [...]

2.2.3.1. In analoger Anwendung der vorstehend skizzierten Rechtsprechung ist zu prüfen, ob Art. 319 StGB, zu dessen Begehung der Beschuldigte B. als Haupttäterin angestiftet hat, in seinem Unrechtsgehalt über Art. 305 StGB hinausgeht beziehungsweise, ob das Entweichenlassen von Gefangenen gegenüber der Begünstigung den Schutz von anderen Rechtsgütern beinhaltet.

2.2.3.2. Art. 305 StGB fällt unter den siebzehnten Titel des Strafgesetzbuches «Verbrechen und Vergehen gegen die Rechtspflege». Geschütztes Rechtsgut von Art. 305 StGB ist das Funktionieren der Strafrechtspflege. [...] Demgegenüber findet sich Art. 319 StGB unter dem achtzehnten Titel «Strafbare Handlungen gegen die Amts- und Berufspflicht». Diese Strafnorm schützt das Interesse der Allgemeinheit am korrekten Funktionieren des Justizwesens, was im engeren Sinne auch, aber nicht nur, das korrekte Funktionieren der Strafverfolgung und des Strafvollzuges beinhaltet [...]. Die unter dem 18. Titel des StGB eingereihten Strafnormen wollen nämlich ins- besondere auch das eminente öffentliche Interesse an der rechtsgetreuen Ausübung öffentlich-rechtlicher Befugnisse durch deren Träger schützen. Mit Strafe bedroht werden unter diesem Titel deshalb verschiedene Arten von ungetreuer und missbräuchlicher Führung öffentlicher Ämter [...]. Wie die Vorinstanz zutreffend ausgeführt hat, zeigt demnach die Systematik des Strafgesetzbuches deutlich, dass mit Art. 319 StGB auch bzw. gar in erster Linie - das Interesse an der getreuen und von Missbrauch freien Führung öffentlicher Ämter geschützt wird. Damit einher geht der Schutz des Vertrauens der Bevölkerung in die Integrität der mit einer öffentlichen Aufgabe betrauten Personen. Der Schutz dieser Interessen geht über den Schutzzweck der Strafrechtspflege im Sinne von Art. 305 StGB hinaus. Während mit Art. 305 StGB die Strafrechtspflege von äusseren rechtswidrigen Einwirkungen geschützt werden soll, will Art. 319 StGB das Justizwesen vor innerem Machtmissbrauch schützen. [...]

13 Die durch Art. 305 und Art. 319 StGB geschützten Rechtsgüter erweisen sich [...] als nicht deckungsgleich. Das Unrecht, das mit Art. 319 StGB begangen wird, erschöpft sich nicht in der Begünstigung des Gefangenen, dem zur Flucht verholfen wurde, sondern besteht darüber hinaus in einem Amtsmissbrauch (im weiteren Sinne) der mit der Bewachung von Gefangenen betrauten Personen und der damit einhergehenden Erschütterung des besonderen Vertrauens des Staates und der Bevölkerung in die Integrität solcher Beamten. [...]

14 Der Strafgrund der Anstiftung liegt in der Mitwirkung an dem vom Haupttäter be- 
gangenen Unrecht (vgl. BGE 115 IV 230 E. 2). Nach dem vorstehend Gesagten hat der Beschuldigte, indem er B. dazu anstiftete, ihn entweichen zu lassen, nicht nur sich selbst begünstigt. Vielmehr hat er über diese Selbstbegünstigungsabsicht hinaus B. bewusst und gewollt zu einem Missbrauch ihrer amtlichen Befugnisse verleitet und damit (vergleichbar zur vorgenannten Rechtsprechung betreffend Art. 286 StGB) in deren konkrete Amtstätigkeit eingegriffen. Der vom Beschuldigten mitgeschaffene Unrechtsgehalt übersteigt denjenigen einer Begünstigung nach Art. 305 StGB und geht damit auch über den Umfang einer straflosen reinen Selbstbegünstigung hinaus. Dass seinem Handeln das Motiv der Selbstbegünstigung zu Grunde lag und eine strafbare Begünstigung objektiv nicht eintrat, stellt unter diesen Umständen keinen Rechtfertigungsgrund für die von ihm begangene Straftat dar (sondern lediglich einen Strafminderungsgrund).

Der Entscheid gibt Anlass zu Bemerkungen in Bezug auf die Anstiftung (IV.), aber auch hinsichtlich der Frage, ob nicht doch eine straflose Selbstbegünstigung vorliegt (V.).

\section{Anmerkungen zur Anstiftung}

16 Der gegen Hassan Kiko erhobene Vorwurf lautet auf Anstiftung zum Entweichenlassen eines Gefangenen (Art. 24 Abs. 1 i.V.m. Art. 319 StGB), wobei vorliegend die Besonderheit besteht, dass der Gefangene der der Anstiftung Beschuldigte selbst ist. In materieller Hinsicht ist zunächst der Frage nachzugehen, ob Hassan Kiko Angela Magdici zu Art. 319 angestiftet hat.

\section{Bestimmen}

17 Das Gesetz umschreibt die Anstiftungshandlung in Art. 24 StGB (lediglich) mit einem «Zur-Tat-Bestimmen». Unbestritten ist dabei zunächst der Effekt der Einflussnahme: Durch die Anstiftung muss in einem andern der Entschluss zu einer bestimmten Tat hervorgerufen werden. Es bedarf eines Kausal- bzw. Motivationszusammenhangs zwischen der Beeinflussung des Anstifters und dem Hervorrufen des Tatentschlusses beim Täter. Wer also bereits zur Tat entschlossen ist, kann nicht mehr angestiftet werden.7

18 Damit ist aber noch nicht gesagt, mit welchen Mitteln der Anstifter vorzugehen hat. Das Bundesgericht lässt grundsätzlich jede psychische, geistige Beeinflussung bzw. eine unmittelbare Einflussnahme auf die Willensbildung eines andern genügen. Es sei nicht erforderlich, dass beim Anzustiftenden Widerstände überwindet werden müssten. Als Anstiftungsmittel komme daher jedes motivierende Tun in Frage, alles, was im andern den Handlungsentschluss hervorrufen könne. Auch eine blosse Bitte, Anregung oder konkludente Aufforderung sei ein taugliches Anstiftungsmittel. ${ }^{8}$

19 Das Obergericht folgt im Wesentlichen dieser Rechtsprechung. Es hält fest, als Anstiftungsmittel komme «jedes motivierende Tun in Frage, alles, was im andern den Handlungsentschluss hervorrufen kann».9 Das bedarf zumindest der

7 Vgl. zum Ganzen etwa BGE 127 IV 122 E. 2b/aa; Günter Stratenwerth, Schweizerisches Strafrecht, Allgemeiner Teil I: Die Straftat, 4. Aufl., Bern 2011, § $13 \mathrm{~N} 102$.

8 Grundlegend BGE 127 IV 122 E. 2b/aa; zuletzt bestätigt im Urteil des Bundesgerichts 6B_17/2016 vom 18. Juli 2017, E. 2.4.2.

9 E. II.2.1.2. 
Präzisierung. Zwar vertritt das Bundesgericht im Ansatz durchaus ein extensives Anstiftungsverständnis. Entgegen dem, was die obergerichtlichen Erwägungen vermuten lassen, wertet das Bundesgericht jedoch nicht jegliche tatentschlussauslösende Einflussnahme als Anstiftung, sondern nur psychische bzw. geistige. Wer demgegenüber - so das Bundesgericht - lediglich eine Situation schaffe, in der sich ein anderer voraussichtlich zur Verübung einer Straftat entschliesse, sei nicht Anstifter (sog. Tatsachenarrangement). ${ }^{10}$

Im vorliegenden Fall geht es indes nicht um ein Tatsachenarrangement. Das Obergericht erwog, Hassan Kiko habe «durch das mehrmalige und konstante Nachfragen» bei Angela Magdici den Tatentschluss hervorgerufen. ${ }^{11}$ Es gilt daher zu klären, ob eine Nachfrage - selbst wenn wiederholt geäussert - eine Anstiftung darstellt.

\section{Anfrage}

21 In BGE 127 IV 122 hatte das Bundesgericht folgenden Fall zu beurteilen: Der Blick-Journalist Viktor Dammann rief bei der Staatsanwaltschaft des Kantons Zürich an und bat um Auskunft darüber, «ob bestimmte Personen insbesondere wegen Betäubungsmitteldelikten vorbestraft seien $»^{2}$. Weil kein Staatsanwalt abkömmlich war, gelangte Viktor Dammann an eine Verwaltungsassistentin, die ihm die gewünschten Informationen zukommen liess. Die Mitarbeiterin der Staatsanwaltschaft wurde von den kantonalen Gerichten wegen Amtsgeheimnisverletzung (Art. 320 StGB) schuldig

Vgl. etwa BGE 128 IV 11 E. 2a.

E. II.2.1.3.

BGE 127 IV 122 E. 2c. gesprochen. Vor Bundesgericht ging es nur noch um Viktor Dammann: Stellte seine Anfrage an die Verwaltungsassistentin eine Anstiftung zu der von dieser begangenen Amtsgeheimnisverletzung dar? Das Bundesgericht bejahte dies. Durch die Frage sei nicht bloss eine tatprovozierende Situation geschaffen, sondern «die Bitte nach einer Antwort geäussert» 13 worden. Und «ohne Frage hätte es keine Antwort gegeben»14. Prima vista liesse sich daher argumentieren, wenn eine Anfrage ein taugliches Anstiftungsmittel sei, so müsse dies für eine (wiederholt geäusserte) Nachfrage auch - und umso mehr - gelten.

22 Gegen eine solche Sichtweise könnte intuitiv eingewendet werden, dass man ja wohl noch werde fragen dürfen. Jedenfalls akzeptierte Viktor Dammann den Entscheid des Bundesgerichts nicht und erhob gegen ihn Beschwerde beim Europäischen Gerichtshof für Menschenrechte (EGMR) in Strassburg. Mit Erfolg: Der EGMR sah in seiner Verurteilung eine Verletzung des Rechts auf freie Meinungsäusserung (Art. 10 EMRK). ${ }^{15}$ Oder anders ausgedrückt: Ja, man wird noch fragen dürfen! ${ }^{16}$

\section{Nachfragen}

23 Kann das Recht auf freie Meinungsäusserung auch Hassan Kiko in Anspruch nehmen? Eine Gemeinsamkeit mit BGE 127 IV 122 liegt darin, dass hier wie dort eine Anstiftung zu Sonderdelikten zur Debatte steht: Amtsgeheimnisverletzung

13 BGE 127 IV 122 E. 2b/bb.

14 BGE 127 IV 122 E. 2c.

15 Urteil des EGMR 77551/o1 vom 25. April 2006 (Dammann gegen Schweiz).

16 Wobei sich diese Aussage naturgemäss nur auf den dem EGMR unterbreiteten Sachverhalt beziehen kann. 
(Art. 320 StGB) im einen, Entweichenlassen von Gefangenen (Art. 319) im anderen Fall. Im Unterschied zu BGE 127 IV 122 handelt es sich bei Hassan Kiko nicht um einen Journalisten, sondern um einen Häftling. Gelten für ihn andere Regeln? Oder ist auch die (wiederholt geäusserte) Nachfrage von Hassan Kiko mit Blick auf Art. 10 EMRK vor einer strafrechtlichen Reaktion geschützt?

BGE 127 IV 122 hat in der (Strafrechts-) Lehre zahlreiche kritische Reaktionen hervorgerufen. ${ }^{17}$ So ist etwa vorgeschlagen worden, den Anwendungsbereich der Anstiftung dahingehend einzuschränken, als die Beeinflussung des Anstifters stets eine Aufforderung zur Tat enthalten müsse. ${ }^{18}$ Bei einer Aufforderung handelt es sich um eine auf Überzeugung gerichtete (persuasive) Beeinflussung. Die Aufforderung hat somit nicht (bloss) informativen, sondern (auch) appellativen Charakter. Blosse Ratschläge, Hinweise

17 Vgl. hierzu eingehend Micha Nydegger, Zurechnungsfragen der Anstiftung im System strafbarer Beteiligung, Zürich 2012, S. 277 ff. Der bundesgerichtlichen Rechtsprechung (nach wie vor) zustimmend: Andreas Donatsch/Brigitte Tag, Strafrecht I, Verbrechenslehre, 9. Aufl., Zürich 2013, S. 155 ff.; Marc Forster, in: Marcel Alexander Niggli/Hans Wiprächtiger (Hrsg.), Strafrecht I, Basler Kommentar, 3. Aufl., Basel 2013, (fortan: BSK StGB I-Bearbeiter) N 16 zu Art. 24 StGB; Kurt Seelmann/Christopher Geth, Strafrecht, Allgemeiner Teil, 6. Aufl., Basel 2016, Rz. 435; Stefan Trechsel/Marc Jean-Richard, in: Stefan Trechsel/Mark Pieth (Hrsg.), Schweizerisches Strafgesetzbuch, Praxiskommentar, 3. Aufl., Zürich/St. Gallen 2018, N 4 zu Art. 24 StGB; Stefan Trechsel/Peter Noll/Mark Pieth, Schweizerisches Strafrecht, Allgemeiner Teil I: Allgemeine Voraussetzungen der Strafbarkeit, 7. Aufl., Zürich/Basel/Genf 2017, S. 207.

18 Vgl. etwa Stratenwerth (Fn. 7), § 13 N 101; Günter Stratenwerth/Wolfgang Wohlers, Schweizerisches Strafgesetzbuch, Handkommentar, 3. Aufl., Bern 2013, N 4 zu Art. 24 StGB; Bernhard Sträuli, in: Robert Roth/Laurent Moreillon (Hrsg.), Code pénal I, Art. 1-110, Commentaire romand, Basel 2009, N 24 zu Art. 24 StGB. auf günstige Tatgelegenheiten und dergleichen würden damit aus dem Anwendungsbereich der Anstiftung ausgeschieden. ${ }^{19}$ Dieser Lösungsansatz zielt zwar in die richtige Richtung, geht aber letztlich $\mathrm{zu}$ wenig weit, wie denn gerade auch BGE 127 IV 122 zeigt. So lässt sich nämlich die Anfrage von Viktor Dammann durchaus als Aufforderung verstehen, ${ }^{20}$ hatte dieser doch ein Interesse, im Rahmen seiner beruflichen Recherchearbeiten an die gewünschten Informationen zu gelangen. Bei objektiver Betrachtungsweise war für die Verwaltungsassistentin deshalb erkennbar, dass in der Anfrage der Wunsch enthalten war, die entsprechenden Informationen zu erhalten. Die Anfrage von Viktor Dammann wäre somit auch nach dieser Auffassung als Anstiftung zu werten und der Widerspruch zur EMRK bliebe bestehen.

\section{Verbindlichkeit}

25 Man wird deshalb als anstiftungsrelevante Motivierung mehr als eine blosse Aufforderung verlangen müssen. So legt zum einen bereits eine unbefangene Lesart des Gesetzestextes nahe, dass mit der Umschreibung «Zur-Tat-Bestimmen» eine gewisse Intensität der Einflussnahme zum Ausdruck gebracht wird. Zum andern gilt zu beachten, dass auf den Anstifter die volle Täterstrafe Anwendung

$19 \overline{\text { Das legt bereits der allgemeine Sprachgebrauch }}$ nahe: Die Redensart, wonach man jemandem durch sachdienliche Hinweise $\mathrm{zu}$ einer Tat verhilft, deutet bereits terminologisch die Nähe zur Gehilfenschaft an.

20 So im Grundsatz auch BGE 127 IV 122 E. 2b/bb («[d]urch die Frage wird vielmehr der Wunsch, die Bitte nach einer Antwort geäussert»); gl.M. Franz Riklin, Anstiftung durch Fragen, GA 2006, S. 361 ff., 363. Entscheidend ist im Übrigen nicht die Form der Äusserung, sondern der Sinngehalt; vgl. Sträuli (Fn. 18), N 25 zu Art. 24 StGB. 
findet. ${ }^{21}$ Insofern können nur solche Beeinflussungen als Anstiftung in Frage kommen, die wesentlichen Einfluss auf den Entschluss des Täters haben. ${ }^{22}$

Voraussetzung für eine anstiftungsgeeignete Aufforderung muss sein, dass sie ein Mindestmass an Verbindlichkeit in sich trägt. An BGE 127 IV 122 verdeutlicht: Viktor Dammann hat der Verwaltungsassistentin weder Vorteile bei kooperativem Verhalten noch Nachteile bei unkooperativem Verhalten explizit in Aussicht gestellt. Es liesse sich zwar argumentieren, jede Aufforderung sei von der Erwartung getragen, dass ihr Folge geleistet werde. Komme der Adressat der Aufforderung nicht nach, so sei er (zumindest) mit der enttäuschten Erwartungshaltung des Auffordernden konfrontiert. ${ }^{23} \mathrm{Er}$ befinde sich insofern in einer dilemmatischen Situation, als er sich zwischen der Begehung der Straftat und der Erwartungsenttäuschung entscheiden müsse. Dem ist jedoch entgegen zu halten, dass die Verwaltungsassistentin Viktor Dam-

21 Das Gesetz sieht in Art. 24 Abs. 1 StGB lediglich eine prinzipielle Gleichstellung von Anstifter und Täter vor. Da die Strafzumessung schuldbezogen und damit individuell vorzunehmen ist (vgl. Art. 27 StGB), ist es im konkreten Einzelfall möglich, dass der Anstifter eine schwerere, dieselbe oder eine leichtere Strafe erhält. Der Grundsatz der limitierten Akzessorietät (vgl. hierzu Stratenwerth (Fn. 7), § 13 N 86) lässt eine strafbare Anstiftung sogar dann zu, wenn sich der Täter mangels schuldhaftem Handeln nicht strafbar gemacht hat.

22 Ähnlich Matthias Schwaibold, Das Auskunftsbegehren als strafrechtliche Anstiftung nach Schweizer Recht. Anmerkung zu einem Entscheid des Schweizerischen Bundesgerichts, AfP 2003, S. 300 ff., 303, der eine «erhebliche Einwirkung auf die Willensbildung» fordert.

23 Weiterführend dazu Knut Amelung, Die Anstiftung als korrumpierende Aufforderung zu strafbedrohtem Verhalten, in: Andreas Hoyer et al. (Hrsg.), Festschrift für Friedrich-Christian Schroeder zum 70. Geburtstag, Heidelberg 2006, S. 147 ff., 164 . mann nicht kannte und ihr daher vernünftigerweise gleichgültig sein musste, falls die Verweigerung der Informationsherausgabe bei ihm eine Erwartungsenttäuschung ausgelöst hätte. Angesichts dessen lässt sich nicht sagen, der Aufforderung von Viktor Dammann komme verbindlicher Charakter zu. ${ }^{24}$ Im Übrigen ist darauf hinzuweisen, dass die Anfrage nicht bereits deshalb anders zu behandeln ist, weil sie von einem Journalisten stammte. ${ }^{25}$

\section{Dilemma}

27 Was den vom Obergericht beurteilten Fall anbelangt, ist zunächst festzuhalten, dass die wiederholte Nachfrage von Hassan Kiko als Aufforderung anzusehen ist, zumal angesichts seiner Situation und der wiederholten Äusserung der «Nachfrage» der Wunsch nach Freilassung klar erkennbar war und Hassan Kiko Angela Magdici für sein Vorhaben hat gewinnen wollen. So geht denn auch das Obergericht von einer «Bitte» ${ }^{26}$ und damit von einer persuasiven Einflussnahme aus. Im Unterschied etwa zur Ausgangslage in BGE 127 IV 122 bestand zwischen Anstifter und Täterin, so das Obergericht, ein «enges persönlichen Verhältnis»»7. In diesem Kontext erfährt die als Aufforderung zu wertende, wiederholte Nachfrage bzw. Bitte ein deutlich anderes Gewicht. Angela Magdici wollte einerseits - aus

24 Näheres bei Nydegger (Fn. 17), S. 316 ff.

25 Im Grundsatz auch Felix Bommer, Anstiftung und Selbstverantwortung, plädoyer 3/02, S. 34 ff., 39; a.M. wohl BSK StGB I-Forster (Fn. 17), N 16 zu Art. 24 StGB.

26 Vgl. E. II.1.2.

27 Medienberichte lassen uns wissen, dass die beiden Beteiligten mittlerweile geheiratet haben. Vgl. etwa Limmattaler Zeitung online vom 11. Juli 2017 (Heirat hinter Gittern: Ex-Gefängnisaufseherin gibt Hassan Kiko das Ja-Wort in Lenzburg). 
menschlich nachvollziehbaren Gründen - die enge persönliche Beziehung zu Hassan Kiko nicht aufs Spiel setzen, indem sie seinem Wunsch nicht nachgekommen wäre. Zudem war ihr ebenfalls daran gelegen, mit ihm in Freiheit ein gemeinsames Leben führen zu können. ${ }^{28}$ Andererseits war ihr bewusst, dass das Verhelfen zur Flucht disziplinar- und strafrechtliche Konsequenzen nach sich ziehen würde.

Angela Magdici befand sich mithin in einem ernsthaften Dilemma, was die Nachfrage bzw. Bitte von Hassan Kiko in einem anderen Licht erscheinen lässt als die Anfrage von Viktor Dammann an die Verwaltungsassistentin. Die Wirkung der Einflussnahme von Hassan Kiko auf Angela Magdici wurde dadurch noch verstärkt, dass er sie über Monate hinweg immer wieder darum bat, ihm zur Flucht zu verhelfen (das Obergericht spricht von «konstanten Bitten» bzw. von «mehrmalige[n] und konstante[n] Nachfragen»). Vor diesem Hintergrund fällt es denn auch nicht schwer, Hassan Kiko als treibende Kraft anzusehen und ihm - gerade auch deshalb - grundsätzlich die volle Täterstrafe zukommen zu lassen. Sein Beitrag zur Entschlussfassung von Angela Magdici war so gesehen ein wesentlicher. ${ }^{29}$ Dem Obergericht ist deshalb im

28 Zur «excusable aspiration à la liberté» s. unten Fn. 42.

29 Verlangt man als Anstiftung einen wesentlichen Beitrag zum Entschluss des Täters (im Sinne einer verbindlichen Tataufforderung), so handelt es sich bei ihm stets um eine qualifizierte Tatveranlassung. Minder intensive Beeinflussungen sind demgegenüber unter die (obligatorisch milder zu bestrafende) Gehilfenschaft nach Art. 25 StGB zu fassen oder haben straflos zu bleiben. Bei dieser Sichtweise wird deutlich, dass der Gedanke des erlaubten Risikos bei der Anstiftung keine (unmittelbare) Bedeutung erlangen kann. Denn mit einer restriktiveren Auslegung des Begriffs «Zur-
Ergebnis darin beizupflichten, dass Hassan Kiko Angela Magdici zur Tat nach Art. 319 StGB «bestimmt» hat.

\section{Sonderdelikt}

29 Der vorliegend zu besprechende Fall zeigt schliesslich, dass die Ausgestaltung der Straftat als Sonderdelikt keinen Einfluss auf die Frage haben kann, ob ein «Zur-Tat-Bestimmen» anzunehmen ist. 30 $\mathrm{Ob}$ der Aufforderung die entscheidende Verbindlichkeit zukommt oder nicht, hängt nicht von der Struktur des Delikts, sondern von der Intensität der Einflussnahme ab. Dabei ist - wie gezeigt - namentlich auch der Kontext mitzuberücksichtigen, in dem sich die Kommunikation zwischen Anstifter und Täter abspielt. Aufgrund der engen persönlichen Beziehung zwischen Hassan Kiko und Angela Magdici war letztere hin- und hergerissen, wie sie sich entscheiden sollte. Gemäss dem Urteil des Obergerichts lehnte sie die Bitte von Hassan Kiko anfänglich ab, kam ihr dann aber doch nach, nachdem sie offenbar über Monate hinweg wiederholt geäussert wurde. ${ }^{31}$ Das Dilemma bestand für Angela Magdici unabhängig davon, ob von ihr die Begehung eines Allgemein- oder eines Sonderde-

Tat-Bestimmen» werden von vornherein nur (erhöht) strafwürdige Tatveranlassungen vom Tatbestand der Anstiftung erfasst; eine Aussonderung «neutraler» Alltagshandlungen erübrigt sich. Den Gesichtspunkt des unerlaubten Risikos bei der Anstiftung berücksichtigend Bommer (Fn. 25), S. 35; Guido Jenny, Die strafrechtliche Rechtsprechung des Bundesgerichts im Jahre 2001, ZBJV 140 (2004), S. 1 ff., 5; Andreas Noll, Ist die an BGE 127 IV 122 geübte Kritik berechtigt?, AJP 12 (2003), S. 892 ff., 899 ff.; Riklin (Fn. 20), S. 364; Stratenwerth (Fn. 7), § 13 N 101; Sträuli (Fn. 18), N 21 zu Art. 24 StGB.

30 A.M. Bommer (Fn. 25), S. 38; Noll (Fn. 29), S. 893 und 897; Riklin (Fn. 20), S. 363 f.

31 Nach den Sachverhaltsfeststellungen des Obergerichts hatte der Beschuldigte "penetrant» bei der Gefängnisaufseherin nachgefragt (E. II.2.1.3). 
likts gefordert wurde. ${ }^{22}$ Insofern stellt die Anstiftung zu einem Sonderdelikt auch kein Sonderproblem dar; die Einflussnahme muss so oder anders das Mass einer verbindlichen Tataufforderung erreichen.

30 Im Übrigen wird der Umstand, dass Extraneus und Intraneus einen unterschiedlichen Bezug zum tangierten Rechtsgut haben, bereits durch die Regelung in Art. 26 StGB abgegolten: Wem die dem Sonderdelikt zugrunde liegende Pflicht nicht zukommt, wird milder bestraft. Diesen für Hassan Kiko entlastenden Umstand hat das Obergericht bei der Strafzumessung denn auch berücksichtigt. 33 Nur bedingt nachsichtig war das Obergericht dagegen, was das Motiv von Hassan Kiko zur Anstiftung betraf: Nämlich die Wiedererlangung der Freiheit als Ausprägung der Selbstbegünstigung. Darauf ist im Folgenden einzugehen.

\section{Anmerkungen zur Selbstbegünstigung}

31 Nebst der Frage, ob die Einwirkung Hassan Kikos auf Angela Magdici als tatbestandsmässige Anstiftung einzustufen ist, lässt sich im vorliegenden Fall vor allem auch darüber streiten, ob sein Verhalten entgegen der Ansicht des Obergerichts nicht doch als (straflose) Selbstbegünstigung zu werten ist. 34 Müsste man

$32 \overline{\text { Wer das anders sieht, schreibt Angela Magdici als }}$ Gefängnisaufseherin letztlich die Rolle eines «emotionslosen Vollzugsorgans» zu. Gerade der Fall des Obergerichts zeigt, dass dies an der Realität vorbeigeht.

33 Vgl. E. III.1.3.2.1.

34 Z.R. bejahend Andreas Eicker, Ist es strafbar, wenn ein Inhaftierter die Aufseherin anstiftet, ihn zu befreien?, plädoyer $5 / 17$, S. 17; ferner für die h.L. zu $\S 120 \mathrm{StGB} / \mathrm{D}$, welcher sowohl die Gefangenenbefreiung durch Private (Art. 310 Ziff. 1 $\mathrm{StGB} / \mathrm{CH}$ ) als auch durch Amtsträger (Art. 319 das Urteil des Obergerichts für Laien zusammenfassen, könnte man das etwa wie folgt tun:

1. Es ist keine Straftat, aus der Haft zu fliehen.

2. Straflos ist man jedoch immer nur solange, als niemand «Halt!» ruft.

3. Fragt man einen Komplizen, ob er einen bei der Flucht hilft, bleibt man straflos.

4. Fragt man hingegen eine Wärterin, ob sie einen aus der Haft entlässt, begeht man eine Straftat.

32 Der erste Punkt betrifft die Frage, weshalb die Selbstbegünstigung straflos zu bleiben hat (1.). Im zweiten und dritten Punkt geht es um die Frage, ob nur die sog. reine Selbstbegünstigung straflos sein soll (2.). Der letzte Punkt betrifft die zentrale Frage, wer strafrechtlich die Verantwortung dafür trägt, Ausbrüche zu verhindern (3.).

\section{Straflose Flucht?}

33 Nicht wenige Laien würden bei der geschilderten Zusammenfassung wohl den Kopf schütteln. Das gilt zunächst für die Straflosigkeit der Flucht. Dass ein Gefängnisausbruch keine Straftat darstellt, 35 sorgt unter Nichtjuristen regel-

StGB/CH) umfasst, Heribert Ostendorf, in: Urs Kindhäuser/Ulfrid Neumann/Hans-Ullrich Paeffgen (Hrsg.), Nomos Kommentar Strafgesetzbuch, 5. Aufl. 2017, N 19 zu § 120; gl.M. für die Teilnahme des Gefangenen an seiner Befreiung durch Private Delnon/Rüdy, in: Marcel Alexander Niggli/Hans Wiprächtiger (Hrsg.), Strafrecht II, Basler Kommentar, 3. Aufl., Basel 2013, (fortan: BSK StGB II-Bearbeiter), N 28 zu Art. 310.

35 So schon die Botschaft vom 23. Juli 1918 zu einem Gesetzesentwurf enthaltend das schweizeri- 
mässig für Erstaunen. ${ }^{36}$ Solchem Volksempfinden gehorchend, reichte SVPNationalrat Lukas Reimann am 19. Juni 2015, also bereits ein halbes Jahr vor den hier interessierenden Ereignissen, eine Motion ein, die verlangte, den «Gefängnisausbruch unter Strafe» zu stellen. Der Bundesrat verwies in seiner ablehnenden Antwort unter anderem auf den Nemotenetur-Grundsatz von Art. 113 StPO, wonach sich niemand selbst belasten müsse. Der Nationalrat verwarf die Motion am 1. Juni 2017 zwar,37 dennoch soll diesem intuitiven Missbehagen hier auf den Grund gegangen werden. Weshalb ist die Selbstbegünstigung straflos?38

sche Strafgesetzbuch (BBl 1918 IV 1); a.M. wohl nur noch Fabio Vassalli, l'evasione nel diritto penale svizzero, Diss. Bern 1967, Mendrisio 1967, S. $91 \mathrm{ff}$.

36 Der Kanton Neuchâtel hatte den Ausbruch (évasion) noch im Jahr 1940 nach kantonalem Strafrecht für strafbar erklärt. Er wähnte sich hierzu nach Art. 335 StGB berechtigt, wurde dann aber von seinem eigenen Kassationsgericht zurückgepfiffen; zum Ganzen François Clerc, de la répression de l'évasion simple (Selbstbefreiung), ZStrR 81/1965, S. 76.

37 Motion (15.3753) vom 19. Juni 2015, Gefängnisausbruch unter Strafe stellen, wurde abgelehnt am 1. Juni 2017 (AB 2017 N 861 f.).

38 Nach François Clerc, de la répression de l'évasion simple (Selbstbefreiung), ZStrR 81/1965, S. 77, soll hier der französische Code pénal den liberalen Strafgesetzgebungen als Vorbild gedient haben; die Straflosigkeit der Selbstbefreiung lässt sich mittelbar sogar aus Art. 180 der Constitutio Criminalis Carolina von 1532 herleiten : « Item so eyn hütter der peinlichen gefengknuß eynem der peinlich straff verwirckt außhilfft, der hat die selbig peinlich straff an statt des übelthätters, den er also ausgelassen, verwirckt. Kem aber der gefangen durch bemelts hütters vnfleiß auß gefengknuß, solcher vnfleiß ist nach gestalt der sachen vnnd radt so an den orten, als hernach gemelt wirdet, zu straffen. »; dazu Hartmut Schneider, Grund und Grenzen des strafrechtlichen Selbstbegünstigungsprinzips auf der Basis eines generalpräventiv-funktionalen Schuldmodells. Diss. Berlin 1990, Berlin 1991, S. 187.
34 Nach Art. 305 Abs. 1 StGB wird wegen Begünstigung bestraft, wer jemanden der Strafverfolgung oder dem Strafvollzug entzieht. Die Selbstbegünstigung bleibt nach ständiger Rechtsprechung und herrschender Lehre jedoch straflos. 39 Das vom Bundesrat angeführte Nemotenetur-Prinzip, also das Recht, sich in einem Strafprozess nicht selber belasten zu müssen, vermag nur zu erklären, weshalb es keine Straftat sein soll, sich einem Strafuerfahren zu entziehen, nicht jedoch, weshalb man sich auch ohne Konsequenzen dem Strafuollzug soll entziehen dürfen.

35 Dass die Selbstbegünstigung straflos sein muss, ergibt sich gemäss dem Bundesgericht bereits aus dem Wortlaut von Art. 305 Abs. 1 StGB. Mit der Wendung, wonach bestraft wird, wer «jemanden» der Strafverfolgung oder dem Strafvollzug entzieht, steht fest, dass der Täter einen andern als sich selbst begünstigen muss. 40

36 Dahinter steckt, wie das Bundesgericht in einem älteren Entscheid ausgeführt hat, die Überlegung, dass straffrei ausgehen soll, wer aus «Selbstschutztrieb» handelt. ${ }^{41}$ Obwohl das Bundesgericht diese

39 BGE 124 IV 127 E. 3b/aa; statt vieler BSK StGB II-Delnon/Rüdy (Fn. 34), N 11 zu Art. 305.

40 BGE 124 IV 127 E. 3b/aa. Dass die Selbstbegünstigung straflos ist, hätte der Gesetzgeber dadurch deutlicher zum Ausdruck bringen können, dass er nicht von «jemanden», sondern von einem «anderen» spricht; vgl. i.d.S. § 258 StGB/D.

41 BGE 73 IV 237; Selbsterhaltungstrieb und Selbstbegünstigung hat im Kontext der Folter bereits der italienische Aufklärer Geatano Filangieri, la scienza della legislazione, Vol. II, Firenze 1782, S. 87, thematisiert: «La prima legge della natura è quella che ci obbliga alla conservazione della propria esistenza. Se richiesto dal magistrato sulla verità dell'accusa, che si è controdi me intentata, io fossi nell' obbligo di confessargli il mio delitto, e se questa confessione mi portasse alla morte, io 
Straffreiheit nicht näher erläutert, legt es ihr wohl Schuldüberlegungen zugrunde: Es soll niemandem zumutbar sein, sich einem Strafverfahren freiwillig zu unterwerfen, und es soll niemandem ein Vorwurf gemacht werden, der versucht, sich einer Strafe zu entziehen. Das Waadtländer Kassationsgericht spricht insoweit eingängig von einer «excusable aspiration à la liberté» ${ }^{2}$, also von einem entschuldbaren Streben nach Freiheit. Aufgrund des Wortlauts von Art. 305 Abs. 1 StGB wäre es jedoch deutlich plausibler, die Selbstbegünstigung als tatbestandslos einzustufen. 43

37 Wenn es dem Gefangenen aber jedenfalls nicht zum Vorwurf gereichen soll, wenn er sich seiner Fesseln entwindet, dann stellt sich natürlich unweigerlich die Frage, wie weit eine solche exceptio libertatis gehen kann.

\section{Reine Selbstbegünstigung}

38 Wie eingangs angedeutet ist die Selbstbegünstigung nicht in allen Fällen straflos. So darf man sich zwar von einem nicht im Strafvollzug einsitzenden Komplizen helfen, sich nicht aber auf der Flucht erwischen lassen. Auch diese Differenzierung dürfte einem Laien nur schwer zu vermitteln sein. Es geht hier um die Frage, in welchem Umfang Selbstbegünstigungen straflos sind.

mi troverei in questo caso tra due doveri opposti e non potrei soddisfare all'uno senza violare l'altro. Se il patto sociale mi obbligasse a questa confessione, il patto sociale mi obbligherebbe a violare una legge anteriore della natura; il patto sociale sarebbe nullo».

42 Cour de cassation du Canton de Vaud vom 2. März 1970, JDT $1970 \mathrm{IV}, 155$.

43 So für die h.L. in D Heribert Ostendorf (Fn. 34), $\mathrm{N} 1$ zu $\S 120$.
39 Das Obergericht hält dazu zunächst fest, dass sich nicht nach Art. 305 Abs. 1 StGB der Begünstigung strafbar macht, wer sich selber der Strafverfolgung oder dem Vollzug einer Sanktion entzieht. 44 Das Gleiche gilt gemäss Obergericht, wenn der Verfolgte/Verurteilte eine Drittperson anstiftet, ihn zu begünstigen, oder die Drittperson ihm bei der Selbstbegünstigung hilft. Letzteres ergibt sich aus BGE 115 IV 230, wo das Bundesgericht eine Verurteilung wegen Anstiftung zur Begünstigung (Art. 24 Abs. 1 i.V.m. Art. 305 Abs. 1 StGB) aufhob.45 In casu hatte ein Strafgefangener eine Bekannte erfolgreich angestiftet, ihm bei der Flucht aus der Strafanstalt zu helfen: Sie holte ihn mit dem Taxi aus der Strafanstalt ab. Sie machte sich damit wohl - was sich dem Entscheid allerdings nicht entnehmen lässt - der Begünstigung strafbar. Er blieb aufgrund Selbstbegünstigung straflos.

40 Straffrei ist gemäss Obergericht - man fühlt sich an Hans Kelsen erinnert - nur die «reine Selbstbegünstigung». Damit ist gemeint, dass eine Selbstbegünstigung nie nach Art. 305 StGB strafbar ist. Selbstbegünstigendes Handeln kann jedoch andere Straftatbestände erfüllen. Das leuchtet etwa in folgendem Fall ein, der ebenfalls ein Stück Zürcher Strafjustizgeschichte geworden ist: Am 17. Dezember 1981 brachen insgesamt sechs In-

44 E. II.2.2.2. m.H.a. BGE 124 IV 127 E. 3b/aa.

45 Gemäss der Regeste von BGE 115 IV 230 seien «Teilnahmehandlungen (Anstiftung und Gehilfenschaft) zur Selbstbegünstigung ... straflos»; richtigerweise geht es in diesem Entscheid aber nur um die «Anstiftung zur Begünstigung des Anstifters» (Stefan Trechsel/Mark Pieth, in: dies. (Hrsg.), Schweizerisches Strafgesetzbuch, Praxiskommentar, 3. Auflage, Zürich 2017, N 14 zu Art. 305) und nicht um den auch denkbaren Fall, dass ein Externer einen Insassen anstiftet, $\mathrm{zu}$ flüchten. 
sassen aus der Justizvollzugsanstalt Regensdorf aus. Einer der Flüchtenden, Pierluigi Facchinetti, erschoss dabei einen Vollzugsbeamten (Fritz Jenni) und verletzte einen weiteren (Rudolf Kottmann) schwer. Ebenfalls unter den Flüchtenden war Marco Camenisch, der einem weiteren Aufseher «aus einer Spraydose eine Flüssigkeit ins Gesicht» gesprayt hatte. 46

Der Umstand, dass die Flüchtenden sich mit der Flucht selbst begünstigten, erlaubte ihnen nicht, Individualrechtsgüter Dritter zu beeinträchtigen. Werden bei einer Flucht Türen aufgebrochen, Justizvollzugsbeamte genötigt, bedroht, verletzt oder gar getötet, hat sich der Flüchtende wegen Sachbeschädigung (Art. 144 StGB), Gewalt und Drohung gegen Behörden und Beamte (Art. 285) sowie Körperverletzungs- oder Tötungsdelikten (Art. $111 \mathrm{ff}$. ) $\mathrm{zu}$ verantworten, allenfalls wegen Meuterei (Art. 311) oder sogar Diebstahls (Art. 139) der Anstaltskleidung47. Facchinetti wurde wegen Mords an Jenni und Mordversuchs an Kottmann vom Geschworenengericht in Winterthur ${ }^{48}$ zu 17 Jahren Zuchthaus verurteilt,49 Camenisch vom Vorwurf der Mittäterschaft zum (versuchten) Mord hingegen freigesprochen. ${ }^{\circ}$

46 Dazu im Detail Kurt Brandenberger, Marco Camenisch - Lebenslänglich im Widerstand, Basel 2015, S. $95 \mathrm{ff}$.

47 So offenbar die h.L. in D, vgl. Schneider (Fn. 38), S. 189.

48 In Winterthur wurden jene Sitzungen des Geschworenengerichts abgehalten, welche ein besonderes Sicherheitsrisiko bargen. "Das Winterthurer Gerichtshaus war nämlich das einzige im Kanton, das mit dem örtlichen Gefängnis durch einen unterirdischen Gang verbunden war», siehe Winterthur Glossar, Bezirksgebäude I.

49 Brandenberger (Fn. 46), S. 104.

5o Brandenberger (Fn. 46), S. 165.
42 Reine Selbstbegünstigung ist somit nie strafbar. Die Frage ist, ob es ausserhalb von Art. 305 StGB und der Anstiftung dazu keine weiteren Fälle reiner, mithin nicht strafwürdiger Selbstbegünstigung gibt. Das Obergericht verneint dies. Es verweist hierzu auf die Rechtsprechung zur Hinderung von Amtshandlungen: Wer die Flucht vor der Polizei ergreife und diese so an der Erfüllung ihres Auftrags hindere, mache sich nach Art. 286 StGB strafbar. ${ }^{1}$ Das Obergericht stützt sich dabei auf BGE 124 IV 127, wo das Bundesgericht entschied, dass sich der Hinderung einer Amtshandlung schuldig mache, «[w]er sich durch Flucht einer Ausweiskontrolle durch einen Polizeibeamten entzieht, um einer Strafverfolgung zu entgehen»(Regeste). Das Selbstbegünstigungsprivileg von Art. 305 Abs. 1 StGB gelte hier nicht.52 Zur Begründung führt das Bundesgericht aus, dass verschiedene Rechtsgüter auf dem Spiel stünden. Art. 286 StGB diene dem Schutz der öffentlichen Gewalt und Art. 305 dem Schutz der Strafrechtspflege. Die Hinderung einer Amtshandlung stelle

51 E. II.2.2.2.

52 BGE 124 IV 127 E. 3b/bb; in diesem Sinn hatte das Bundesgericht bereits 1959 im (wegen der dort ebenfalls behandelten Hehlerei-Frage) legendären Coq-de-bruyères-Fall (BGE 85 IV 142) entschieden, wo es um Auerhahn-Wilderer im Chasseral ging, die sich durch Flucht einem Gendarmen entzogen, der den Kofferraum ihres Wagens kontrollieren wollte: «Il est exact que celui qui se soustrait lui-même à une poursuite pénale ou à l'exécution d'une peine ne tombe pas sous le coup de l'art. 305 CP (RO 73 IV 239 c. 1). Cela ne signifie cependant pas qu'il bénéfice nécessairement de l'impunité. Son acte peut en effet constituer une autre infraction... et tel est en particulier le cas lorsque la fuite a pour effet - voulu par le fuyard - d'empêcher l'agent d'accomplir l'acte qui lui incombe. Ainsi, le condamné qui prendrait la fuite pour échapper au policier chargé de le mener au pénitencier et l'empêcherait, ce faisant, de remplir sa mission, encourrait la peine prévue par l'art. 286 CP.» (E. 2). 
daher ein anderes Delikt dar. Solche weiteren Straftaten seien nicht durch Selbstbegünstigung $\mathrm{zu}$ rechtfertigen. Die Selbstbegünstigung dürfe nicht als «Freibrief verstanden werden, jegliche Art von Amtshandlungen, insbesondere solche von Strafuntersuchungsbehörden, zu erschweren oder gar zu verunmöglichen»53.

Die Rechtsprechung argumentiert somit konsequent, man könnte auch sagen: stur, mit den Rechtsgütern. Einzig und allein das von Art. 305 StGB geschützte Rechtsgut (Schutz der Strafrechtspflege) soll dem Selbstbegünstigungsprivileg unterliegen. Das überzeugt wie erwähnt in Bezug auf Individualrechtsgüter. Niemand soll sich ungestraft den Weg aus der Haftanstalt freischiessen dürfen. Ebenso überzeugend ist umgekehrt, dass der Schutz der Strafrechtspflege nicht Sache der Beschuldigten und Verurteilten sein kann. Weder der Beschuldigte, der sich mit den Zwängen eines Strafverfahrens konfrontiert sieht, noch eine Verurteilte, die gegen ihren Willen im Strafvollzug sitzt, können unter Strafe verpflichtet werden, für das reibungslose Funktionieren der Strafrechtspflege einzustehen.

44 Bereits deutlich weniger einleuchtend ist, weshalb der letztgenannte Gedanke nicht auch für die Hinderung einer Amtshandlung gelten soll. Nach der Rechtsprechung darf niemand, um das eingangs genannte Beispiel aufzugreifen, vor einer angedrohten Verhaftung fliehen. Hier hat man den Eindruck, dass die Rechtsprechung die Geister, welche sie mit der Anerkennung des Selbstbegünstigungsprivilegs bei Art. 305 StGB rief, wieder los-

53 BGE 124 IV 127 E. 3b/bb. werden wollte. Obwohl es auch bei der Flucht um den «Selbstschutztrieb» geht, greife das Selbstbegünstigungsprivileg nicht, weil ein anderes Rechtsgut betroffen sei. In Art. 305 gehe es um den Schutz der Rechtspflege, Art. 286 diene dem Schutz der öffentlichen Gewalt.

45 Man kann sich bereits fragen, ob hier phänomenologisch wirklich unterschiedliche Rechtsgüter betroffen sind und ob nicht die öffentliche Gewalt auch die Rechtspflege umfasse, zumal es in beiden Fällen darum geht, den Staat in seinem Wirken nicht zu behindern. 54 Jedenfalls aber ist die strikte Rechtsgüter-Argumentation zu formalistisch. Bei Art. 305 StGB herrscht Einigkeit, dass der Schutz der Strafrechtspflege nicht Sache des Beschuldigten/Verurteilten sein kann. Bei Art. 286 soll er aber für die öffentliche Gewalt einstehen müssen. Hier wird der Betroffene plötzlich zum Garanten für das reibungslose Funktionieren von Amtshandlungen. Es ist ihm bei Strafe verboten, des Amtsschimmels ungestörten Ausritt zu stören. Wenn man nicht die jeder Widersetzung gegen Amtshandlungen innewohnende laesio maiestatis zum entscheidenden Kriterium erklären will, 55 gibt es keinen nachvollziehbaren Grund, die unbemerkte (Art. 305) und die bemerkte (Art. 286) Flucht unterschiedlich zu behandeln. Anders zu entscheiden bedeutete, dass man zwar fliehen darf, aber nur solange niemand

54 Gerhard Fiolka, Das Rechtsgut, Band 2: Das Rechtsgut in Aktion, Dissertation Fribourg 2006, Basel 2006, S. 744.

55 Ähnlich Schneider (Fn. 38), S. 188, wonach «der Pönalisierung der Selbstbefreiung ein autoritäretatistischer Zug eigen» ist. 
«Halt!» ruft. Das kann man keinem Nichtjuristen erklären. 56

\section{Verantwortung}

46 Der obergerichtliche Entscheid würde unter Laien wohl auch deshalb für Kopfschütteln sorgen, weil er in sich nicht kohärent ist: Wenn es schon keine Straftat darstellen soll, auf eigene Faust oder gar mit Hilfe von Komplizen aus dem Gefängnis zu türmen, weshalb soll es dann strafbar sein, die zuständige Wärterin höflich zu fragen, ob sie einen gehen lässt oder gar mitkommt?

Das Obergericht erklärt dies mit einer Analogie. Es wendet die skizzierte Rechtsprechung zur reinen Selbstbegünstigung analog auf Art. 319 StGB, das Entweichenlassen von Gefangenen, an. Die Begünstigung nach Art. 305 schütze das Funktionieren der Rechtspflege. In Art. 319 gehe es nicht nur um das Funktionieren der (Straf-)Justiz, sondern auch um das Vertrauen der Öffentlichkeit in die Justiz. Als Amtsdelikt verbiete Art. 319 nicht nur die Begünstigung des Gefangenen, sondern sanktioniere auch den Amtsmissbrauch der mit der Bewachung von Gefangenen betrauten Personen. $\mathrm{Zu}$ verhindern gelte es die «Erschütterung des besonderen Vertrauens des Staates und der Bevölkerung in die Integrität solcher Beamten». An der Schlüsselstelle führt das Obergericht sodann aus: Während mit Art. 305 die Strafrechtspflege von äusseren rechtswidrigen Einwirkungen geschützt werden

56 So z.R. Günter Stratenwerth/Felix Bommer, Schweizerisches Strafrecht, Besonderer Teil II: Straftaten gegen Gemeininteressen, 7. Auflage, Bern 2013, N 12 zu § 52. soll, wolle Art. 319 das Justizwesen vor innerem Machtmissbrauch schützen. 57

48 Bereits im vorangegangenen Absatz wurde aufgezeigt, dass die RechtsgüterArgumentation des Obergerichts $\mathrm{zu}$ formalistisch ist. Das wird hier noch augenscheinlicher, zumal das in Bezug auf die Selbstbegünstigung entscheidende Rechtsgut der ungestörten Strafrechtspflege identisch ist. Sogar das Obergericht räumt ein, dass die Begünstigung nach Art. 305 StGB und das Entweichenlassen Gefangener nach Art. 319 beide das reibungslose Funktionieren der Justiz und deren Freiheit vor «äusseren» Einwirkungen schützen. Um eine reine und damit strafbefreiende Selbstbegünstigung dennoch verneinen zu können, erklärt das Obergericht kurzerhand das zweite Rechtsgut des Vertrauens in die Strafrechtspflege für entscheidend. Das Justizwesen müsse vor «innerem» Machtmissbrauch geschützt werden. Dass es Amtsmissbrauch im Allgemeinen und die Freilassung von Strafgefangenen im Besonderen unter allen Umständen zu verhindern gilt, wird niemand ernsthaft bestreiten wollen. In der Tat würde das Vertrauen in die Justiz massiv leiden, wenn gegen Polizeigewalt oder Gefangenenbefreiung nicht konsequent vorgegangen würde.

49 Die entscheidende Frage ist aber: Wer ist für diesen Vertrauenserhalt verantwortlich? Wer muss unter Strafandrohung dafür einstehen, dass Beamte ihre Macht nicht missbrauchen? Die Antwort des Obergerichts ist hier: Der von der amtlichen Machtausübung direkt betroffene Gefangene. Das kann nicht richtig sein. Genauso wenig wie Beschuldigte/Verur-

57 E. II.2.2.3.2. 
teilte für das reibungslose Funktionieren der Strafrechtspflege einstehen müssen, sind sie Garanten für das Vertrauen in die Strafjustiz. ${ }^{8}$ Der Staat selbst hat dafür zu sorgen, dass seine Beamten ihre Befugnisse nicht missbrauchen. Tun sie es dennoch, dann muss der Staat seine Beamten zur Verantwortung ziehen. Um es in den Worten des Obergerichts auszudrücken: Das «Justizwesen vor innerem Machtmissbrauch»59 zu schützen, liegt in der Verantwortungssphäre des Staates und nicht des Bürgers. ${ }^{60}$

5o Dass nicht Private für das deliktsfreie Funktionieren des Staatsapparates zur Verantwortung gezogen werden sollen, ist der Zürcher Strafjustiz bereits einmal von höchster Stelle angemahnt worden. Der Blick-Journalist Viktor Dammann wurde vom Zürcher Obergericht wegen Anstiftung zur Amtsgeheimnisverletzung verurteilt, weil er bei der Staatsanwaltschaft Informationen zu Vorstrafen der mutmasslichen Täter des Fraumünster-Postraubs verlangt hatte. ${ }^{61} \mathrm{Hi}-$ erzu hielt der Europäische Gerichtshof für Menschenrechte fest:

«La Cour... estime au contraire qu'il appartient aux Etats d'organiser leurs services et de former leurs agents de sorte qu'aucun renseignement ne soit divulgué concernant des

58 Vgl. schon François Clerc, de la répression de l'évasion simple (Selbstbefreiung), ZStrR 81/1965, S. 77.

59 E. II.2.2.3.2.

60 Zur Frage, ob man den Bürger, der hier zwar in Selbstbegünstigungsabsicht gehandelt hat, aber immerhin eine Beamtin «bewusst und gewollt $\mathrm{zu}$ einem Missbrauch ihrer amtlichen Befugnisse verleitet» hat, für diese Korruption der Beamtin nach den Grundsätzen der Anstiftung strafrechtlich zur Verantwortung ziehen kann, vgl. oben IV.

61 Dazu eingehend BGE 127 IV 122; vgl. bereits oben bei Fn. 12. données considérées comme confidentielles. Ainsi, le gouvernement défendeur assume, en l'espèce, une partie importante de la responsabilité pour l'indiscrétion commise par l'assistante du parquet du canton de Zurich» 62 .

51 Auch nach der Strassburger Rechtsprechung muss somit nicht der Private dafür einstehen, dass Beamte ihre Amtspflichten nicht verletzen, vielmehr ist der Staat dafür verantwortlich.

52 Der Gefangene, welcher eine Wärterin bittet, ihn freizulassen, sabotiert nicht das Vertrauen in die Justiz, sondern reklamiert seine «excusable aspiration à la liberté». Triebfeder ist der Selbstschutz, es liegt mithin reine Selbstbegünstigung vor. Zusammengefasst ist es nicht nur erlaubt, eine Komplizin zu bitten, einen im Taxi aus dem Strafvollzug abzuholen, sondern auch eine Wärterin zu bitten, das Taxi zu bestellen.
$6 2 \longdiv { \text { Urteil des EGMR 77551/o1 vom 25. April } 2 0 0 6 }$ (Dammann gegen Schweiz), Ziff. 55. 